\title{
COCAST: MULTICAST MOBILE AD HOC NETWORKS USING COGNITIVE RADIO
}

\author{
Wooseong Kim, Soon Y. Oh and Mario Gerla \\ Computer Science Department \\ University of California, Los Angeles USA \\ Email: \{wooseong,soonoh,gerla\}@cs.ucla.eud
}

\author{
Joon-Sang Park \\ Department of Computer Engineering \\ Hongik University, Seoul Korea \\ Email: \{jsp\}@hongik.ac.kr
}

\begin{abstract}
Many multicast protocols have been proposed for ad hoc networks. ODMRP is one of the most popular protocols due to its robustness in highly mobile wireless networks. However, ODMRP is unscalable in terms of the number of sources. We improve the scalability of ODMRP using cognitive radio technology, which diversifies channel usage for multicast members with multiple cognitive channels. We design a channel allocation and distribution scheme to build a multicast tree for each group. In addition, we implement cooperative sensing by multicast members using Join Query and Join Reply messages. In simulation experiments, our protocol, CoCast, demonstrates a superior delivery ratio and throughput performance to conventional ODMRP.
\end{abstract}

\section{INTRODUCTION}

In today's warfare, communication between soldiers is considered to play an important role in the success of mission operations. In particular, multicast communication is widely used in tactical field operations to facilitate effective coordination between group-oriented actions undertaken by military units. For such environments, wireless mobile ad hoc networks (MANETs) have received considerable attention as tactical field communication schemes since they are self-organizing wireless network composed of sets of cooperative mobile participants. The communication devices in these networks are small in size and reasonably priced; furthermore, they consume less power and can be procured offthe-shelf without requiring any infrastructure support. However, radio communications in tactical MANETs are exposed to external interference (e.g., jamming by adversaries) and internal interference (e.g., radio channel congestion due to scalability problems).

Spectrum diversity is a good countermeasure against jamming and congestion, since it exploits multiple radios or channels to increase capacity and scalability, as well as to protect communication against jamming attacks and congestion. Upon detecting jamming or channel congestion, it switches to other empty channels/frequencies on the basis of monitored channel information. Many cognitive radio protocols have been proposed in the literature, but they deal with only unicast communication in wireless networks. Therefore, they are not applicable to tactical MANET scenarios, where multicast communication is used for accomplishing team-based missions. Therefore, in this study, we propose a new multicast routing protocol using a cognitive radio system, called CoCast, which alleviates the jamming and scalability problem by employing a cognitive radio system.

In 1999, J. Mitola [8] proposed a cognitive radio based on Software Defined Radio (SDR) [7], which fully utilizes empty channel holes without interrupting licensed users or primary users. According to the Federal Communications Commission (FCC), licensed spectrums are operated at only $15 \% \sim 85 \%$ regionally. Based on these figures, the FCC released its Notice of Proposed Rule Making (NPRM) [1] for cognitive radio in 2003, which allows secondary users to access licensed bands using cognitive radio technology. In addition, the IEEE 802.22 Wireless Regional Area Network (WRAN) [2] standardizes a specification to realize cognitive radio in current wireless networks. Even though many cognitive radio researches have been conducted, from the physical layer to the application layer, multicast communication using cognitive radio is still an unexplored region. Most researches focus on channel sensing/detecting, media access control (MAC), and network layer unicast routing protocol design, or theoretical studies on achievable capacity or efficient channel assignment schemes.

CoCast is inspired by the On-Demand Multicast Routing Protocol (ODMRP) [5] design. First, all of the nodes in multicast groups cooperatively detect other signals, periodically sensing their energy and exchanging this channel sensing information. This channel information is piggybacked on Join Query and Join Reply messages, which travel on a predefined common channel, and nodes select an appropriate channel that 
can avoid jamming and congestion. Moreover, maximum channel utilization among multicast groups must be considered to maximize data throughput and optimize the multicast route. Join Query and Reply are periodically exchanged to refresh the multicast route, e.g., every three seconds, and CoCast updates channel assignments based on new channel information during a route refreshing period to adapt to dynamic channel conditions. We assume that nodes in the network have a single radio transceiver that requires a channel switching delay whenever changing channels.

The rest of this paper is organized as followed: Section 2 discusses related works and Section 3 describes the details of the proposed protocol. Section 4 presents simulation results and Section 5 concludes this paper.

\section{RELATED WORK}

In this section, we briefly review previous MANET multicast protocols and existing wireless routing protocols that use a cognitive radio system.

\subsection{Ad Hoc Multicast Protocols}

Over the last few years, many ad hoc multicast routing protocols have been proposed. Some of these protocols use a proactive mechanism that periodically exchanges routing information to maintain the route, while on-demand multicast protocols exchange routing information only when it is necessary. Thus, ondemand routing achieves significant advantages by alleviating a scalability problem in MANET environments.

On-demand routing protocols usually employ twoway handshaking to find a route between a sender and a receiver. The sender floods a request and each receiver sends back a reply packet. To reduce packet flooding, some protocols introduce a local recovery approach. In other words, a local alternative route to the destination is searched when a node detects route breakage. Adaptive Demand-Driven Multicast Routing (ADMR) [3] and the Multicast Ad hoc On-demand Distance Vector (MAODV) [11] protocol are two prominent examples of on-demand ad hoc multicast protocols that follow this approach.

ODMRP [5] is another popular ad hoc on-demand multicast routing protocol that employs a routing mesh instead of a routing tree. It relies on periodic networkwide flooding for route mesh discovery and maintenance. Periodic mesh refreshing and redundant data forwarding in the mesh ensure a higher packet delivery ratio and robustness against mobility and unreliable wireless link propagation, but give rise to scalability problems due to high packet overheads in the network.
To alleviate packet overhead, Enhanced ODMRP (EODMRP) [9] employs dynamic adaptive route refresh and local recovery.

\subsection{Protocols Using Cognitive Radio}

Khalife et al. proposed a routing protocol where nodes estimate channel capacity considering interference from primary and secondary users and exchange probabilistic capacity information using advertisement messages globally [4]. Then, a source node calculates the shortest path to the destination using Dijkstra's algorithm considering the required data rate from an application. However, the authors did not consider the channel switching delay and deafness problem in cognitive radio networks. STOD-RP [15] is an ondemand routing protocol with a tree structure. It divides the intra-spectrum and inter-spectrum routings in cognitive radio networks. Each node acts like a normal ad hoc network node for intra-spectrum routing, but in inter-spectrum routing, intermediate nodes are used as switch nodes. STOD-RP can reduce the channel switching delay by domain separation and control packet flooding restrictions. Pyo et al. focused on neighbor discovery and route establishment in cognitive wireless networks [10]. They introduced Common Link Control Radio (CLCR) as a common active wireless system for cognitive terminals and developed a Minimum Weight Routing Protocol (MWRP), which selects a minimum communication overhead route. In [14], all of the channels are layered and a node selects a path to a destination using a conflict graph for each layer; however, the problem with this approach is that it does not clearly account for the dynamic changes in networks. The SPEctrum-Aware Routing Protocol (SPEAR) [12] integrates the end-to-end optimization of flow-based approaches with the flexibility of link-based approaches. Further, Ma et al. proposed a spectrum aware routing protocol [6] that weights the cost of edge with delay without using a common control channel. The above protocols are applied to wireless ad hoc routing, but no study to this day has proposed a MANET multicast routing protocol using cognitive radio.

\section{Cocast}

In this section, we present a detailed description of CoCast.

\subsection{Channel Scanning}

Efficient spectrum sensing is one of the main challenges for CoCast. Generally, for spectrum sensing, a transceiver compares the received signal energy level to 
the receiving sensitivity or detects a specific signal feature from incoming signals, e.g., pilot signals, cyclic prefixes, symbol rates, spreading codes, modulation types, etc. In a tactical MANET, cognitive radio channel scanning presents several challenges. First, it is difficult to find the proper channel scanning rate. Cognitive radio must periodically scan the channels that it wants to use. Frequent channel scanning reduces the chance of interruption of primary users, but it decreases throughput. Further, the synchronization between nodes during the quiet period, which is the channel scanning period, is another critical challenge in an ad hoc cognitive radio system. Last, since a tactical field operation can potentially cover a large area and obstacles exist that might block the signal, different nodes have different available channel views. To overcome the above problems, CoCast employs cooperative sensing, where nodes share scanned channel information with neighboring nodes to avoid channel conflicts.

In CoCast, if a node is switched on, it initially scans its own spectrum during the cold start period and builds a channel table that contains the noise level, expected capacity, center frequency, validity, etc. This channel information is propagated via a predefined common channel that is piggybacked on control packets during multicast route establishment and refreshment periods. These data are used for channel selection between nodes. The channel scanning duration is determined as follows:

$$
\begin{gathered}
D_{\text {total }}=D_{h w}+D_{\text {protocol }} \\
D_{h w}=D_{R F_{-} \text {Rec }}+D_{B B_{-} R e c} \\
D_{\text {protocol }}=D_{\text {Ch_access }}+D_{\text {Scheduling }}
\end{gathered}
$$

where $D_{R F_{-} R e c}$ is the $\mathrm{RF}$ reconfiguration delay, $D_{B B_{-} R e c}$ is the baseband reconfiguration delay, $D_{\text {Ch_access }}$ is the channel access delay, and $D_{\text {Scheduling }}$ is the channel scheduling delay. In general, the RF reconfiguration delay is considered to be $125 \mu$ s and the baseband reconfiguration delay is estimated to be 24 ms. The channel access delay is uniformly distributed over a period of $100 \mathrm{~ms}$ in the multicast case, and the scheduling delay varies on the basis of the number of forwarding channels. After channel scanning, each node manages channel sets as follows:

Scanned channel set, $A s=\left\{C_{0}, C_{1}, C_{2}, C_{3}, \cdots, C_{n}\right\}$ Available channel set, $A c=\left\{C_{0}, C_{1}, C_{6}, C_{7}\right\}$
Active channel set, $A a=\left\{C_{1}, C_{6}\right\}$

A scanned channel set is a list of channels that are scanned during the cold start period, and a node gets an available channel set by filtering the occupied channels. The node selects an active channel set from among the available channel set for packet transmitting. The reason why a node picks multiple channels as the active set is to reduce channel switching delay. A node chooses active channels randomly or selects a set of adjacent channels. In the above example, we used random selection for the active channels and the same in the simulation. The node monitors the in-band signal strength of the active channel set during data transmission. If the signal noise ratio decreases below a defined threshold, the node removes the channel from the active channel set and moves to another active channel or a common channel.

\subsection{Multicast Route Establishment and Chan- nel Allocation}

The process of multicast route creation and maintenance in CoCast is the same as that of ODMRP. When a source has packets to send, it starts broadcasting a Join Query packet via a predefined common channel. Upon receiving the non-duplicated Join Query packet, a node records the upstream node address in the routing table to learn the reverse path and rebroadcasts it. When the Join Query reaches a multicast member node, the node creates a Join Reply packet and sends it toward the source. The Join Reply packet is relayed through the learned reverse path and the nodes on the reverse path are organized as a "forwarding group." After exchanging the Join Query/Reply, the multicast route is created and data is delivered through this multicast route.

During the route establishment period, CoCast executes channel allocation. A node writes its own active channel list on the Join Query packet. Upon receiving the active channel list from an upstream node, the node selects a listening channel from the received channel list. To select the listening channel, the node compares the received channel list with its own active channels and then with its own available channels if there is no common channel in its own active channel set. If the node fails to find an overlapped channel in its own available channel set, the node ceases Join Query broadcasting. When a node rebroadcasts the Join Query, it removes the upstream node's active channels and writes its own active channel list. Upon receiving the Join Query, a receiver node writes the selected listening channel on 
the Join Reply and sends it toward the source. Nodes on the reverse path also overwrite the listening channel on the Join Reply. However, if it is possible, forwarder nodes adjust the listening channel based on the downstream node's listening channel to synchronize the channel in the multicast route.

If the number of assigned channels for a multicast session increases, the throughput deteriorates because the channel switching delay increases. To minimize this delay, CoCast tries to assign a single channel for each multicast tree. However, the heterogeneity of the available channels in each node prevents the unification of the allocated channels for the tree. Forwarder nodes may switch a few different channels to relay data. If the number of forwarder nodes increases, the channel switching delay may increase, since more channels can be assigned. Therefore, to reduce the number of forwarders, CoCast routing uses the tree structure instead of the mesh style; thus, it cannot exploit the data packet redundancy advantage of the mesh structure.

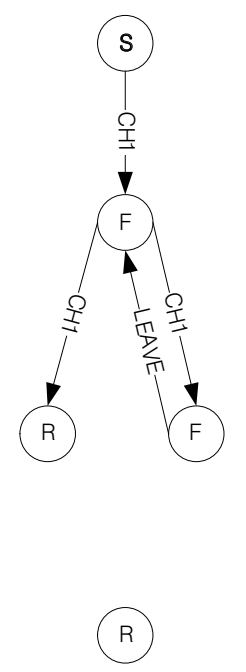

(a)

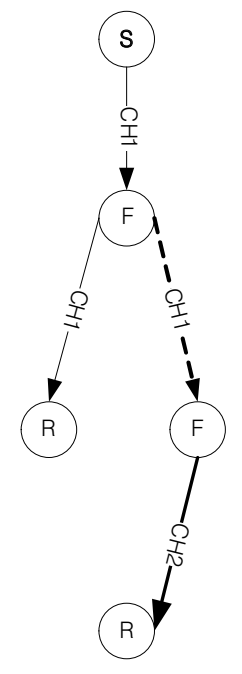

(b)
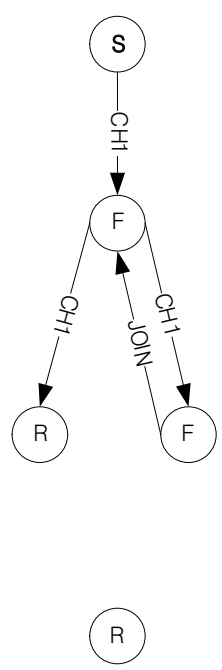

(c)

\section{Figure 1. Multiple forward nodes for channel switching.}

\subsection{Data Transmitting}

After establishing the route, a source starts transmitting data in the network via the forwarding group. Nodes in the forwarding group receive and relay data packets over selected channels. A forwarder node tries to synchronize the listening channel with the downstream node's listening channel to reduce channel switching delay, but multiple downstream nodes may select different channels. Moreover, if the upstream node and the downstream node select different chan- nels, the forwarder node must use different listening and transmitting channels. In this case, the forwarder must repeatedly switch channels to receive and relay data packets. It is therefore possible for the forwarder to miss packets, since the upstream node may transmit packets while the forwarder node switches to a different channel to relay packets. This is called the deafness problem, and it occurs in the case of nodes with a single transceiver. CoCast avoids the deafness problem by adding LEAVE and JOIN control packets. In 1 (a), the forwarder node sends the LEAVE message to the upstream node after receiving a packet. In this case, the upstream node stops relaying packets for as long as the downstream node is unavailable and the downstream forwarder node can transmit a packet by switching from channel 1 to channel 2 , as seen in 1 (b). When the downstream forwarder node returns to the listening channel, $\mathrm{CH} 1$, it sends the JOIN message to the upstream node. The forwarder node transmits LEAVE and JOIN packets only when it is using multiple channels for receiving and transmitting; otherwise, it is switched off.

\subsection{Route Refresh}

Since the multicast tree in a MANET is very fragile due to node mobility, many MANET protocols use local route recovery when route failure occurs. However, detecting route breakage is a difficult challenge in MANET multicast. Thus, like ODMRP, CoCast employs periodic route refresh. CoCast exchanges Join Query/Reply messages periodically, e.g., every $3 \mathrm{~s}$ in our simulation, allowing it to rebuild the multicast tree to adapt to dynamic topology changes in the MANET. CoCast does not employ a time synchronization scheme or special control packets for synchronizing route refresh. A source node propagates the route refresh rate and route refresh period interval written on the Join Query packet. We only assume that all of the nodes have their own clock and calculate the next route refresh time and refresh period individually without time synchronization.

During the route refresh period, nodes switch to the common channel and remain on that channel. The route refresh process is the same as the route establishing process. A source node broadcasts a Join Query packet and receivers respond with a Join Reply packet. During the route refresh period, channels for the multicast session are reassigned. When transmitting Join Query and Reply packets, nodes update the active channel list and embed the active list and the listening channel in the packets. To properly update the active 
channel list, a node must rescan all the channels, but scanning the entire channel spectrum requires too much time. Thus, CoCast only monitors the in-band energy levels of active channels. If the node senses other signals on the active channels, it scans other available channels to find candidate active channels. Every node periodically rescans all of the channels with a very low rate, e.g., every $100 \mathrm{~s}$.

This route refresh process is the same as that of ODMRP, but unlike ODMRP, CoCast does not employ a forwarder lifetime. ODMRP's forwarder lifetime is several times longer than the route refresh interval, so that the number of forwarders and the extent of redundant packet forwarding increase with time. However, CoCast's forwarder lifetime is the same as the route refresh interval. If a forwarder node misses a Join Reply after it relays a Join Query, the node is freed from the forwarding group. Thus, a CoCast multicast route bears a greater resemblance to a tree structure than to a mesh structure.

\section{SIMULATION RESULT}

This section presents the simulation setup and results. In accordance with previous studies, we implement CoCast using Qualnet [13], a packet level network simulator, and compare its performance with that of ODMRP.

\subsection{Simulation Setup}

We implement a simple cognitive radio system in Qualnet, in which both channel scanning and switching are available. The cognitive radio system has an effective reception range of $376 \mathrm{~m}$ and a channel capacity of 2 Mbps. For a single-channel ODMRP, we use $802.11 \mathrm{~b}$ with the same radio range and channel capacity. The packet size is 512 bytes and the traffic is 4 packets/s. One hundred nodes are randomly distributed in a $2000 \mathrm{~m} 2000 \mathrm{~m}$ field and a Random Waypoint mobility model is applied to mobile nodes with a maximum node speed of $20 \mathrm{~m} / \mathrm{s}$ and a pause time of zero. We use the Qualnet default values for the other layer configuration and parameters.

The CoCast and ODMRP refresh intervals are 3 s and the CoCast forwarder lifetime is the same as the refresh interval, while the ODMRP forwarder lifetime is 3 times the refresh interval. The CoCast route refresh process lasts $500 \mathrm{~ms}$ (so nodes stay on the common channel for $500 \mathrm{~ms}$ ) and a node scans all of the channels every 100 s.

For performance evaluation, we use two metrics: the Packet Delivery Ratio, which is the fraction of received packets, and the Average End-to-End Delay, which is the average time taken for a packet to be transmitted across the network from a source to a destination. One simulation lasts 300 simulation seconds and all of the numbers are averaged over 100 simulation runs.

\subsection{Varying Number of Sources}

In this subsection, we present and analyze our simulation results when the number of sources is varied. We assume that nodes have 7 available channels and that the number of sources in a multicast session increases from 2 to 10 , while the multicast session has 30 receivers. We run the simulation with mobility (ODMRP_Mob and CoCast_Mob) and without mobility.

Figure 2 shows the delivery ratios of CoCast and ODMRP when the number of sources is varied. When the number of sources increases, the packet delivery ratios of CoCast and ODMRP decrease since the network congestion increases. However, the rates of decrease are significantly different. When there are more than 4 sources, the ODMRP delivery ratio decreases rapidly, indicating that this is the point at which the network becomes saturated. However, the CoCast delivery ratio decreases slowly, since CoCast distributes flows over multiple channels and can alleviate congestion for each channel. Mobility adversely affects both protocols, and thus the delivery ratios decrease when the nodes move. However, the delivery ratio decrease rate in ODMRP is bigger than that in CoCast, even though ODMRP uses a forwarding mesh structure. Since the forwarder lifetime is 3 times longer than the refresh interval in ODMRP, the forwarding mesh becomes larger with time. Thus, congestion is more serious due to redundant packet forwarding in the mesh.

Figure 3 shows the end-to-end delay when the number of sources is varied. We provide the same broadcast jitter to both protocols, e.g., $10 \mathrm{~ms}$. As the number of sources increases, the ODMRP end-to-end delay rapidly increases. After network saturation, it is difficult for nodes to grasp an opportunity to transmit a packet in ODMRP. Therefore, packets stay in the queues of intermediate nodes for a long time. In contrast, CoCast prevents congestion by distributing flows over multiple channels. Thus, CoCast can maintain a low end-to-end delay. As in the delivery ratio case, mobility increases the end-to-end delay since it causes more congestion and link breakage in a network. 


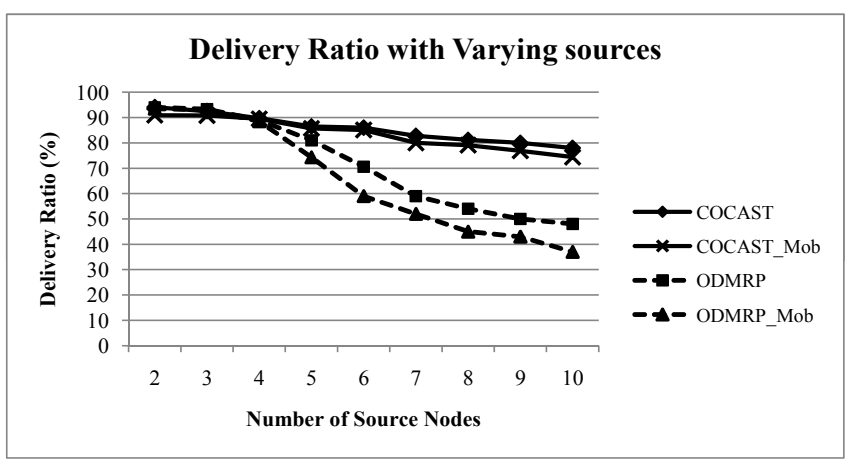

Figure 2. Data delivery ratio when the number of multicast sources is varied.

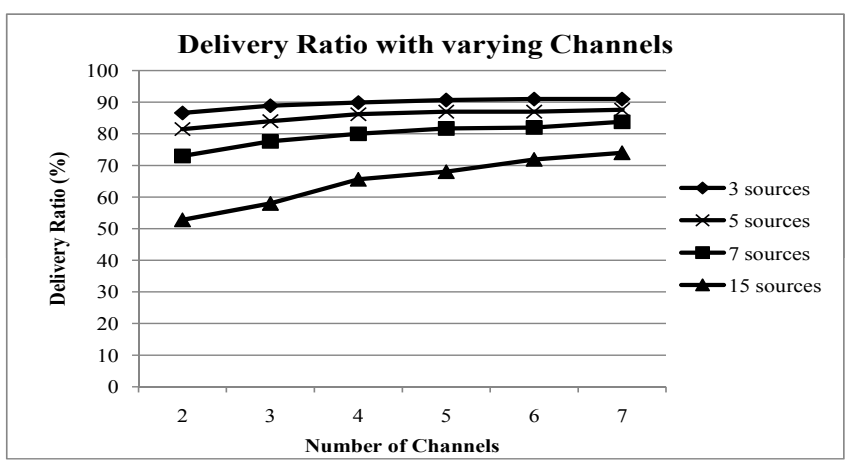

Figure 4. Data delivery ratio when the number of channels is varied.

\subsection{Varying Number of Channels}

Figure 4 and 5 show the performance of CoCast when the number of channels is varied. There is one multicast session and 10 receivers with varying numbers of sources. The nodes have 7 channels and they move based on Random Waypoint mobility model, with a maximum speed of $20 \mathrm{~m} / \mathrm{s}$ and a pause time of zero. Figure 4 shows that the delivery ratio increases slowly for up to 7 sources, since the network congestion is not serious in each channel. The delivery ratio lines increase linearly and become level when the number of channels exceeds the number of sources. In the 15-sources case, serious channel congestion occurs when the number of channels is small, and the delivery ratio line increases linearly. We can observe congestion with 15 sources in Figure 5. Since the congestion is not serious, the end-to-end delay is less than $200 \mathrm{~ms}$ when the number of sources is less than 7. However, heavy traffic from 15 sources causes serious congestion and 7 channels are not enough to solve the traffic congestion problem for 15 sources.

\subsection{Jamming Attack Situation}

One of the important advantages of using CoCast is its ability to foil a jamming attack initiated by an ad-

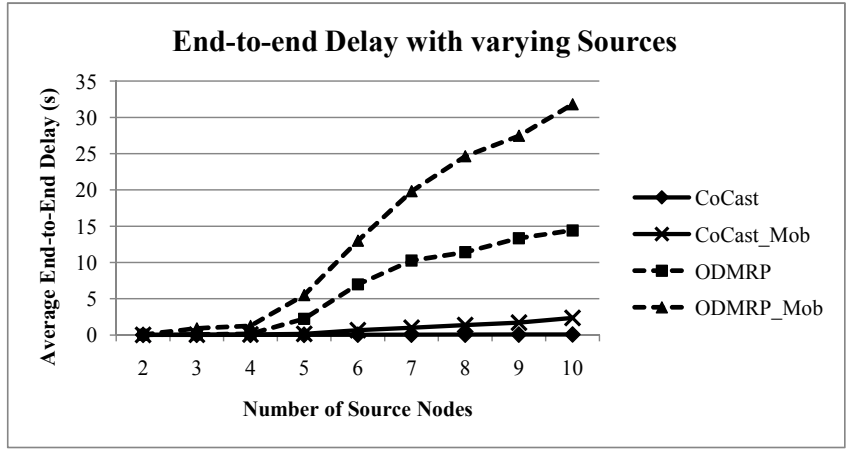

Figure 3. Average end-to-end delay when the number of multicast sources is varied.

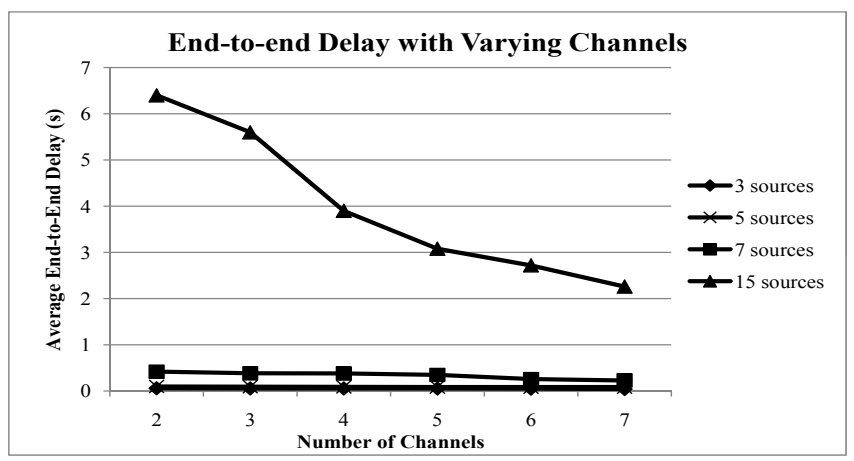

Figure 5. Average end-to-end delay when the number of channels is varied.

versary in a tactical field operation by using its multichannel agility. If CoCast detects jamming or channel congestion, it hops onto another available channel. To validate this, we design a simulation model for a jamming attack. There are 100 moving nodes in a 1500 m $1500 \mathrm{~m}$ battlefield. The nodes begin multicast communication and the adversary initiates a jamming attack upon detecting the communication signals. The receiver nodes cannot receive packets during the attack period if they use the same channel as the adversary. We choose one receiver node and measure its throughput as a function of time.

Figure 6 shows the effect of jamming when we use ODMRP. The selected node has an unstable link since it misses numerous packets at the beginning of the simulation. Its throughput decreases when the jamming attack begins and recovers after the attack ends. That is, ODMRP's throughput is badly affected by a jamming attack. However, CoCast shows considerable robustness against the jamming attack (Figure ??). When CoCast identifies a jamming attack by detecting the energy level of the incoming signal, it quickly moves to another active channel. At around $148 \mathrm{~s}$, its throughput decreases to zero for a few seconds, since it takes time 


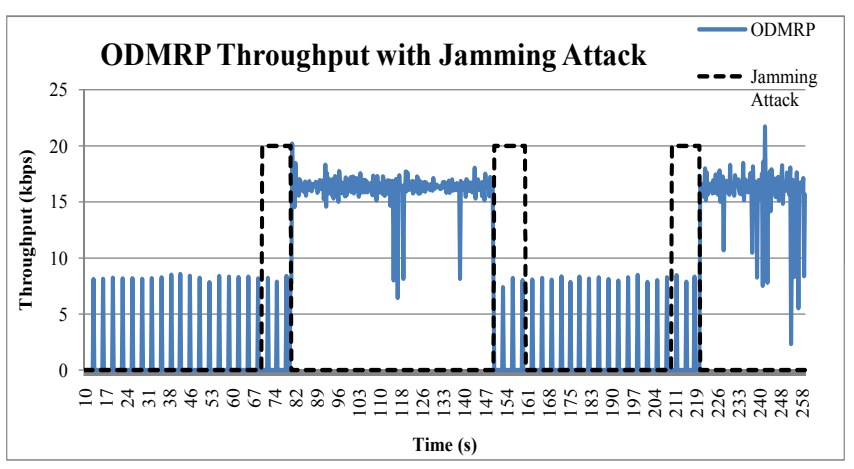

Figure 6. ODMRP's throughput under jamming attack. The dotted line represents the intensity of jamming traffic; the solid line indicates receiver throughput.

to find another available channel, but in other cases it switches to another active channel very quickly, making it difficult to find a throughput drops. Thus, as expected, CoCast is a good solution for coping with jamming attacks in tactical MANET scenarios.

\section{CONCLUSION}

In this paper, we presented CoCast, an ad hoc multicast protocol for cognitive radio based MANETs. CoCast alleviates ODMRP's scalability problem with respect to the number of multicast sources using multiple cognitive radio channels. We have shown via simulation CoCast's scalability with the number of sources in term of delivery ratio, end-to-end delay, and throughput. .

\section{ACKNOWLEDGE}

This research is supported through participation in the International Technology Alliance sponsored by the U.S. Army Research Laboratory and the U.K. Ministry of Defense under Agreement Number W911NF-06-3-0001; and by ARMY MURI under funding W911NF0510246.

\section{References}

[1] $F C C \quad 02-322 \quad N P R M$ on Cognitive Radio. http://hraunfoss.fcc.gov/edocs_public/attachmatch/ FCC-03-322A1.pdf.

[2] IEEE 802ஷ்2. http://www.ieee802.org/22/.

[3] J. G. Jetcheva and D. B. Johnson. Adaptive demand-driven multicast routing in multi-hop wireless ad hoc networks. In MobiHoc '01: Proceedings of the 2nd ACM international symposium on Mobile ad hoc networking $\&$ computing, pages 33-44, Long Beach, CA, USA, 2001.

[4] H. Khalife, S. Ahuja, N. Malouch, and M. Krunz. Probabilistic path selection in opportunistic cognitive radio networks. In GLOBECOM, pages 4861-4865. IEEE, 2008.

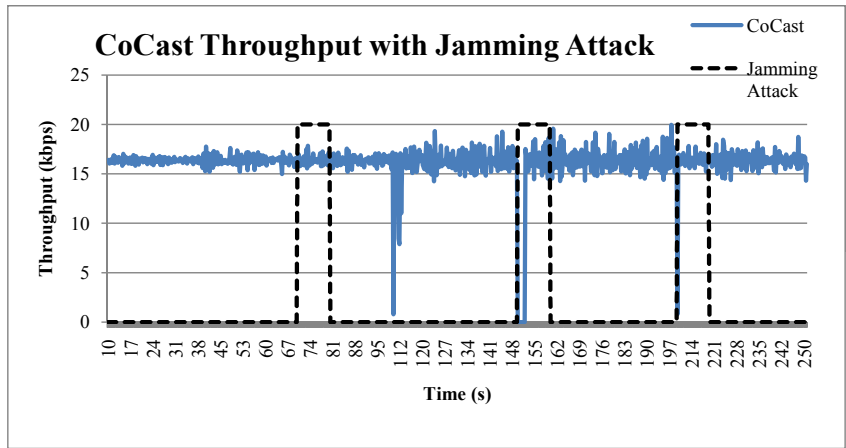

Figure 7. CoCast's throughput under jamming attack. The dotted line represents the intensity of jamming traffic; the solid line indicates receiver throughput.

[5] S. J. Lee, W. Su, and M. Gerla. On-demand multicast routing protocol in multihop wireless mobile networks. Mobile Networks and Applications, 7(6):441-453, 2002.

[6] H. Ma, L. Zheng, X. Ma, and Y. luo. Spectrum aware routing for multi-hop cognitive radio networks with a single transceiver. In International Conference on Cognitive Radio Oriented Wireless Networks and Communications (CROWNCOM 2008), Singapore, May 2008.

[7] J. Mitola. Software Radios: Wireless Architecture for the 21st Century. John Wiley \& Sons, Inc, 2000.

[8] J. Mitola and G. Maguire. Cognitive radio: making software radios more personal. Personal Communications, IEEE [see also IEEE Wireless Communications], 6(4):13-18, 1999.

[9] S. Y. Oh, J. sang Park, and M. Gerla. E-odmrp: Enhanced odmrp with motion adaptive refresh. Journal of Parallel and Distributed System, 64(8):1044-1053, 2008.

[10] C. W. Pyo and M. Hasegawa. Minimum weight routing based on a common link control radio for cognitive wireless ad hoc networks. In IWCMC '07: Proceedings of the 2007 international conference on Wireless communications and mobile computing, pages 399-404, New York, NY, USA, 2007. ACM.

[11] E. M. Royer and C. E. Perkins. Multicast operation of the adhoc on-demand distance vector routing protocol. In The 5th annual ACM/IEEE international conference on Mobile computing and networking (MobiCom '99), Seattle, WA, USA, 1999.

[12] A. Sampath, L. Yang, L. Cao, and H. Z. B. Y. Zhao. High throughput spectrum-aware routing for cognitive radio based ad-hoc networks. In International Conference on Cognitive Radio Oriented Wireless Networks and Communications (CROWNCOM 2008), Singapore, May 2008.

[13] Scalable Networs Inc. QualNet. http://www.scalblenetworks.com.

[14] C. Xin, L. Ma, and C.-C. Shen. A path-centric channel assignment framework for cognitive radio wireless networks. Mobile Networks and Applications, 13(5):463-476, 2008.

[15] G.-M. Zhu, I. F. Akyildiz, and G.-S. Kuo. Stod-rp: A spectrum-tree based on-demand routing protocol for multihop cognitive radio networks. In GLOBECOM, pages 30863090. IEEE, 2008. 